\title{
HADRON SPECTROSCOPY WITH CLAS AND CLAS12*
}

\author{
D.I. GLAZIER \\ Universiy of Glasgow, School of Physics and Astronomy \\ University Avenue, Kelvin Building, Glasgow G12 8QQ, United Kingdom
}

(Received June 15, 2015)

The CLAS detector completed a highly successful data-taking lifetime in 2012. The mass range accessible with the CEBAF $6 \mathrm{GeV}$ electron beam and the large solid angle of the detector made it the premier facility for studying baryon resonances. The analysis of a great number of final states with differing polarization states is underway and will lead to a greater understanding of the existing nucleon resonances. In the meantime, preparation for an upgraded detector, CLAS12, is progressing. With commissioning due to start in 2016 with a $11 \mathrm{GeV}$ electron beam CLAS12 will kinematically favour the study of mesons. In particular, it will look to resolve outstanding questions as to the make-up of mesonic states in terms of hybrid states with gluonic constituents; states of pure glue; or tetraquark/molecular configurations.

DOI:10.5506/APhysPolBSupp.8.503

PACS numbers: 14.20.Gk, 14.40.Rt, 13.60.Le

\section{Introduction}

QCD provides, in principal, a quantitative description of the strong interaction. The strong interaction is responsible for the existence of a spectrum of hadronic states made up from quarks and bound by a gluonic field. Hence, understanding the hadronic spectrum in terms of QCD will provide an important validation of the theory. QCD is numerically very difficult to solve, although much progress is being made via lattice and many other approaches. As a result, we strive to describe the known states in terms of QCD degrees of freedom such as constituent quarks and try to determine a quantitative understanding of the confinement of quarks and gluons. This methodology is benefiting from new insights provided by photoproduction experiments carried out at a number of facilities, all coupling intense and polarised photon beams to high acceptance detector systems.

\footnotetext{
* Presented at "Excited QCD 2015", Tatranská Lomnica, Slovakia, March 8-14, 2015.
} 
One such experiment is the CLAS detector in Hall B of the Jefferson Lab. It recently completed its life-cycle producing large datasets for different photo- and electroproduced states, including many different polarization observables.

\section{Jefferson Lab}

The Thomas Jefferson National Accelerator Facility (JLab) houses the Continuous Electron Beam Accelerator Facility (CEBAF), producing a highcurrent, high-duty factor, longitudinally polarized electron beam feeding three experimental halls. CEBAF is composed of two linear accelerators, each adding $0.4 \mathrm{GeV}$ to the electrons each pass, and two sets of recirculating arcs. The electron beam circulates up to five times in the accelerator, to reach the maximum energy of about $6 \mathrm{GeV}$. Three independent beams, with different energies and currents, can be sent to the three experimental halls. JLab is currently undergoing an upgrade of the accelerator and of the existing experimental halls, while a new experiment, Hall D, has recently been constructed. The accelerator portion of the upgrade will be constructed on the framework of the existing CEBAF accelerator. Five new superconducting radio-frequency accelerating elements will be added to each of the linacs, the existing RF cavities will be increased in gradient to achieve a $1.1 \mathrm{GeV}$ per pass acceleration, and a new recirculating arch will be added to provide an additional pass through the North Linac prior to entering Hall D. This new configuration, Fig. 1, will bring the beam up to $11 \mathrm{GeV}$ for Halls A, B and $\mathrm{C}$, and up to $12 \mathrm{GeV}$ for Hall D.

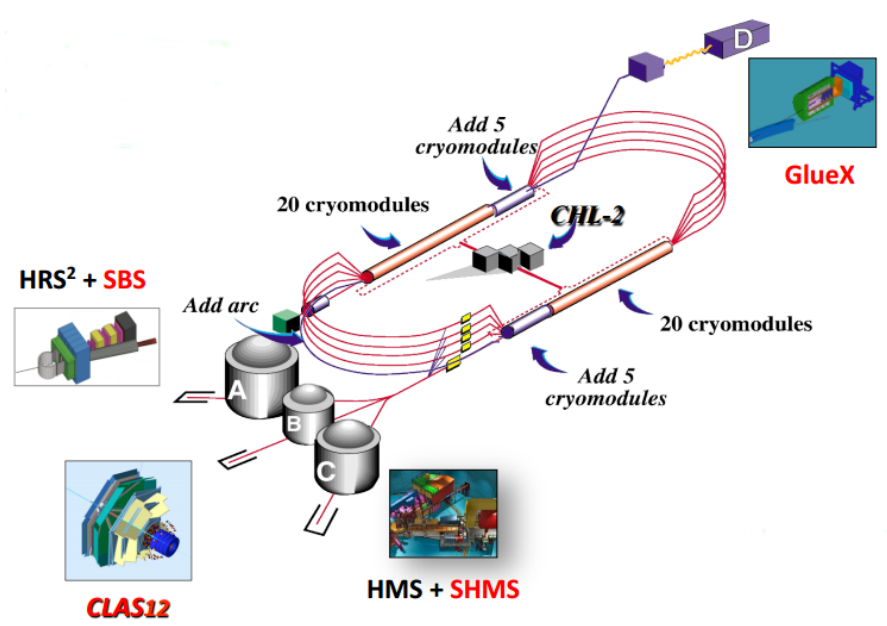

Fig. 1. The upgraded CEBAF accelerator and experiments. 


\section{The CLAS detector}

The main feature of CLAS was 6 superconducting coils producing a nonuniform toroidal magnetic field. Each sector contained three regions of drift chambers that were used to track charged particles and reconstruct their momenta; scintillator counters for particle identification based on time of flight; Čerenkov counters to identify electrons; and electromagnetic calorimeters to measure electrons and neutral particles. Charged particles with laboratory polar angles between $8^{\circ}$ and $135^{\circ}$ were measured with an azimuthal acceptance of around with $83 \%$ and resolutions of 1-mrad polar and 4-mrad azimuthal angle and $0.5 \%$ momentum.

\section{Baryon spectrosopy}

One of the primary physics goals of CLAS was the search for missing resonances. It is a long standing problem that many of the states predicted by the constituent quark model have not been observed in pion scattering experiments. The strategy adopted for this experiment was to photo-produce and measure a large set of final states, including pions but also other pseudoscalar mesons. The production mechanism at these energies is often dominated by the s-channel production of baryonic resonances which subsequently decay via the mesons. The electron beam energy of $6 \mathrm{GeV}$ allowed excitation of nucleon and $\Delta$ resonances up to around $2.4 \mathrm{GeV}$ mass giving access to many of the so-called missing states. To disentangle different contributions, in particular the different s-channel partial waves, to the final state it is necessary to measure polarizations of the beam, target and recoiling nucleon. This was achieved by the CLAS through in a variety of techniques. The longitudinally polarized electron beam transferred circular polarization to the bremsstrahlung produced photon. Coherent bremsstrahlung using an orientated diamond radiator produced linearly polarized photons. The initial nucleon was polarized in frozen spin butanol and HD targets. For the recoil, it was possible to measure the polarization of the $\Lambda$ hyperon through its weak decay, to give a complete set of polarization measurements for kaon photoproduction.

Many such experiments have been already published. For example, the photon asymmetry of the production plane of pions produced with a linearly polarized beam was found to have a significant effect on the couplings of various $\Delta$ states [1]. Measurements of pion production with cirularly polarized beams and longitudinally polarized targets have improved the helicity couplings of resonances [2]. A particularly strong impact has been already made by $K^{+}$production experiments where access to the polarization of the recoil $\Lambda$ has provided measurements of several additional polarization observables prior to runs with polarized targets [3,4]. Partial Wave Analysis including this data has already established the existence of 4 new resonances [5]. 
In addition to this core program, many additional paths were developed. Electroproduction was used to investigate resonances with increasing photon virtuality, providing insight into the nature of several states and, in particular, the Roper resonance e.g. [6,7]. Two-meson production was used to identify cascade decays of resonances which may not decay strongly directly though single pions. Vector mesons such as the $\omega$ and $\phi$ were measured and may couple strongly to particular states e.g. [8]. Neutron targets were also used and are critical to identifying the isospin components of the partial waves and resonances which may couple stronger than for protons. Resonances containing strange quarks, hyperons and cascades have been measured, produced in association with kaons e.g. [9].

This wealth of information provides many different views of baryons and a consistent picture should be required to explain the full set of measurements. So far, the data published is the tip of the iceberg with many publications imminent from the neutron target, polarized target and linearly polarized beam experiments.

\section{The CLAS12 detector}

The new CLAS12 detector for the upgraded $11 \mathrm{GeV}$ CEBAF electron beam will feature several improvements from the original experiment. The luminosity will be an order of magnitude higher; the forward detector will allow for improved particle identification, including neutrals; a silicon-strip/ micromegas hybrid detector will give precise vertex measurements; and a forward electron detector will allow tagging of low $Q^{2}$ virtual photons which contribute a high flux of events into the overall detector. This latter component is designed for and crucial to the meson spectroscopy experiment MesonEx [10].

\section{Meson spectroscopy}

Mesons are the simplest bound system of quarks, being made by a quark and an anti-quark. Therefore, they provide the ideal "laboratory" to study the interaction between quarks, understand the role of gluons, and investigate the origin of confinement. The Constituent Quark Model can be used to predict the existence of multiplets of mesons with similar properties and masses. While most of the lowest mass states have been clearly identified and studied, several open issues remain. Clearly states with more than 2 constituent quarks will be beyond the CQM but not QCD itself, for example, states with constituent gluons (hybrids); tetraquarks; or pure glue (glueballs). In the light quark sector, there has been no definitive identification of such configurations, although there are several candidates particularly in the scalar spectrum. Recent results in the charm quark sector have pointed to strong candidates for tetraquark states making a detailed examination of the light quark sector imperative. 
The goal of the MesonEx experiment is to perform a comprehensive study of the meson spectrum in the light quark sector, in the mass range of a few $\mathrm{GeV}$, with precise determination of resonance masses and properties.

Other experiments share similar ambitions, such as the GluEx experiment in Hall D using a real photon beam and COMPASS using high energy pion beams. Indeed, the COMPASS experiment is already well established and has recently published results pointing to a new axial vector state decaying to three pions via the $f_{0}(980)$ isobar, the $a_{1}(1420)$ [11]. They make an attractive case for this being a tetraquark state and additional states outwith recognised nonets are good evidence for states beyond the CQM. However, others have reported that such a state may be produced via a rescattering effect after the decay of the $a_{1}(1260)$ [12]. It is important to note that with the precision of data generated by this new generation of experiments such higher order effects can become significant. It is, therefore, crucial that the analysis of these datasets incorporates higher order effects.

\section{HASPECT}

The aim of the HASPECT (HAdron SPEctroscopy CenTer) project is to address the need for more elaborate tools in amplitude analysis of high statistics experimental datasets, and MesonEx experiment in particular [13]. All possible theoretical constraints should be respected, such as unitarity, analyticity, crossing symmetry and high/low energy constraints. To assist with this, the group work particularly closely with the Joint Physics Analysis Centre, based at JLab and providing close theoretical support. Many earlier techniques are being revisited, such as longitudinal phase space analysis to kinematically separate different reaction mechanisms. At the amplitude level, Veneziano amplitudes are being developed for use with multiparticle final states [14]. In reality, there is no easy solution and quick feedback between experimentalists and theory will be required on a case-by-case basis to provide an optimal analysis. This will be facilitated through the HASPECT Collaboration.

\section{Conclusions}

The CLAS detector completed a highly successful data taking period in 2012. It has collected high quality data on a number of topics, in particular, photoproduction of baryon resonances to many final states. Polarization observables have been measured and many more are under analysis, with complete sets of experiments feasible for $K^{+} \Lambda$ production. Incorporation of these upcoming results in modern partial wave analysis will help answer many open questions on the baryon spectrum. In the future, the CLAS 
will run again with an upgraded detector optimised for the $11 \mathrm{GeV}$ CEBAF electron beam. This will provide excellent conditions for investigations of mesonic states. High precision results are expected. To help facilitate the analysis of this data, a new collaboration between the experimentalists and theorists has been initiated. The goal is to respect the theoretical constraints inherent in the scattering amplitudes and account for dynamical effect at the percent level or lower. Hopefully, this will allow clear identification of new states.

\section{REFERENCES}

[1] M. Dugger et al., Phys. Rev. C 88, 065203 (2013).

[2] S. Strauch et al., arXiv:1503.05163 [nucl-ex].

[3] R.K. Bradford et al., Phys. Rev. C 75, 035205 (2007).

[4] M.E. McCracken et al., Phys. Rev. C 81, 025201 (2010).

[5] A. Anisovich et al., Eur. Phys. J. A 48, 15 (2012).

[6] V.I. Mokeev et al., Phys. Rev. C 86, 035203 (2012).

[7] K. Park et al., Phys. Rev. C 91, 045203 (2015).

[8] M. Williams et al., Phys. Rev. C 80, 065208 (2009).

[9] L. Guo et al., Phys. Rev. C 76, 025208 (2007).

[10] M. Battaglieri et al., Meson spectroscopy with low $q^{2}$ electron scattering in CLAS12, 2011, unpublished.

[11] C. Adolph et al., arXiv:1501.05732 [hep-ex].

[12] M. Mikhasenko, B. Ketzer, A. Sarantsev, Phys. Rev. D 91, 094015 (2015).

[13] E. Santopinto, J. Phys.: Conf. Ser. 527, 012028 (2014).

[14] A.P. Szczepaniak, M. Pennington, arXiv:1403.5782 [hep-ph]. 